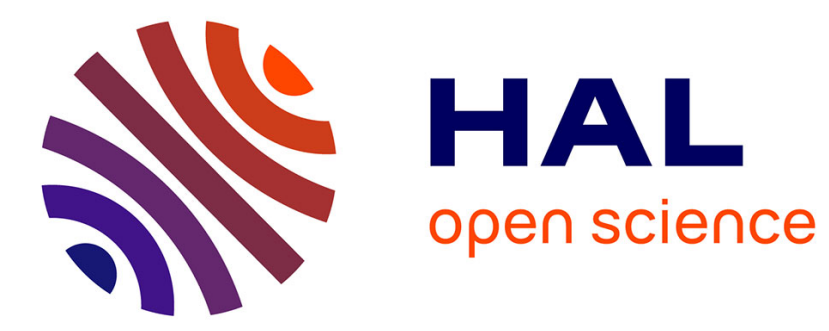

\title{
Complementarity problems with respect to Loewnerian cones
}

\author{
Alberto Seeger, David Sossa
}

\section{To cite this version:}

Alberto Seeger, David Sossa. Complementarity problems with respect to Loewnerian cones. Journal of Global Optimization, 2015, 62 (2), pp.299-318. 10.1007/s10898-014-0230-y . hal-01326152

\section{HAL Id: hal-01326152 \\ https://hal.science/hal-01326152}

Submitted on 1 Nov 2017

HAL is a multi-disciplinary open access archive for the deposit and dissemination of scientific research documents, whether they are published or not. The documents may come from teaching and research institutions in France or abroad, or from public or private research centers.
L'archive ouverte pluridisciplinaire HAL, est destinée au dépôt et à la diffusion de documents scientifiques de niveau recherche, publiés ou non, émanant des établissements d'enseignement et de recherche français ou étrangers, des laboratoires publics ou privés. 


\title{
Complementarity problems with respect to Loewnerian cones
}

\author{
Alberto Seeger · David Sossa
}

\begin{abstract}
This work deals with the analysis and numerical resolution of a broad class of complementarity problems on spaces of symmetric matrices. The complementarity conditions are expressed in terms of the Loewner ordering or, more generally, with respect to a dual pair of Loewnerian cones.
\end{abstract}

Keywords Nonlinear complementarity problem · Loewner ordering · Cone-constrained eigenvalue problem $\cdot$ Semismooth Newton method

\section{Introduction}

The main concern of this work is the analysis and numerical resolution of a class of nonlinear complementarity problems formulated in $\mathcal{S}_{n}$, the space of symmetric matrices of order $n$. As usual, $\mathcal{S}_{n}$ is equipped with the trace inner product $\langle Y, X\rangle=\operatorname{tr}(Y X)$ and the associated norm. The first part of this work deals with a complementarity problem of the form

$$
(\mathrm{SDCP}) \quad\left\{\begin{array}{l}
\Phi(X, Y, \lambda)=\mathbf{0} \\
\mathbf{0} \preceq X \perp Y \succeq \mathbf{0}
\end{array}\right.
$$

David Sossa is supported by CONICYT (Chile).

\section{A. Seeger}

Department of Mathematics, University of Avignon, 33 rue Louis Pasteur, 84000 Avignon, France e-mail: alberto.seeger@univ-avignon.fr

D. Sossa

Departamento de Ingeniería Matemática, Centro de Modelamiento Matemático (CNRS UMI 2807), FCFM, Universidad de Chile, Blanco Encalada 2120, Santiago, Chile

e-mail: dsossa@dim.uchile.cl 
where the nonnegativity constraints are expressed in terms of the Loewner ordering $\succeq$ on $\mathcal{S}_{n}$. Following [20], we refer to the equilibrium model (1) as the Semi-Definite Complementarity Problem (SDCP). The symbol $\lambda$ stands for an unknown parameter vector in a Euclidean space $\Lambda$ and $\Phi$ is a continuously differentiable function from $\mathbb{E}:=\mathcal{S}_{n} \times \mathcal{S}_{n} \times \Lambda$ to another Euclidean space $\mathbb{F}$. The dimension of each bold marked zero vector $\mathbf{0}$ is understood from the context.

Although it is not strictly necessary, for simplicity in the exposition we assume that

$$
\operatorname{dim}(\mathbb{F})=\operatorname{dim}(\Lambda)+\operatorname{dim}\left(\mathcal{S}_{n}\right) .
$$

Such a dimensionality requirement is automatically satisfied in many practical cases. Below we display two concrete examples for which the assumption (2) is in force.

Example 1.1 Let $\operatorname{End}\left(\mathcal{S}_{n}\right)$ denote the vector space of linear endomorphisms on $\mathcal{S}_{n}$. A Loewner-eigenvalue of $\mathfrak{L} \in \operatorname{End}\left(\mathcal{S}_{n}\right)$ is a scalar $\lambda \in \mathbb{R}$ such that the system

$$
\mathbf{0} \preceq X \perp(\mathfrak{L}(X)-\lambda X) \succeq \mathbf{0}
$$

has a nonzero solution $X \in \mathcal{S}_{n}$. Finding a Loewner- eigenvalue of $\mathfrak{L}$ amounts to solve the complementarity problem

$$
\left\{\begin{array}{l}
\mathfrak{L}(X)-\lambda X-Y=\mathbf{0} \\
\operatorname{tr}(X)-1=0 \\
\mathbf{0} \preceq X \perp Y \succeq \mathbf{0} .
\end{array}\right.
$$

The second equality in (3) is a normalization condition which ensures that $X$ is a nonzero solution. In this example one has $\Lambda=\mathbb{R}$ and $\mathbb{F}=\mathcal{S}_{n} \times \mathbb{R}$. Theorem 2.1 in [24] ensures that the system (3) admits always a solution. The problem of finding Loewner-eigenvalues for some particular linear endomorphisms is addressed for instance in [29].

Example 1.2 Consider an optimization problem of the form

$$
\text { minimize } c(X) \text { s.t. } X \succeq \mathbf{0}, \mathcal{A}(X)=b,
$$

where $c: \mathcal{S}_{n} \rightarrow \mathbb{R}$ is a twice continuously differentiable convex function, $\mathcal{A}: \mathcal{S}_{n} \rightarrow \mathbb{R}^{m}$ is a linear map, and $b \in \mathbb{R}^{m}$. A particular instance of (4) is the so-called nearest correlation matrix problem, see $[6,17]$. The KKT-optimality conditions for the minimization problem (4) are

$$
\left\{\begin{array}{l}
\nabla c(X)-\mathcal{A}^{T}(\lambda)-Y=\mathbf{0} \\
\mathcal{A}(X)-b=\mathbf{0} \\
\mathbf{0} \preceq X \perp Y \succeq \mathbf{0},
\end{array}\right.
$$

where $\nabla c: \mathcal{S}_{n} \rightarrow \mathcal{S}_{n}$ is the gradient map of $c$ and $\mathcal{A}^{T}$ is the adjoint map of $\mathcal{A}$. In this example one has $\Lambda=\mathbb{R}^{m}$ and $\mathbb{F}=\mathcal{S}_{n} \times \mathbb{R}^{m}$.

Remark 1.3 Other particular cases of (1) can be found in [7,13,20]. Let $Q \in \mathcal{S}_{n}$ and $\mathfrak{L} \in$ $\operatorname{End}\left(\mathcal{S}_{n}\right)$. The so-called semidefinite linear complementarity problem

$$
\left\{\begin{array}{l}
\mathfrak{L}(X)+Q-Y=\mathbf{0} \\
\mathbf{0} \preceq X \perp Y \succeq \mathbf{0}
\end{array}\right.
$$

also fits into the model (1). Semidefinite LCPs have been analyzed by numerous authors, both from a theoretical and algorithmic point of view. 
The second part of our work deals with a complementarity problem having the more general form

$$
\left\{\begin{array}{l}
\Phi(X, Y, \lambda)=\mathbf{0} \\
\mathcal{K} \ni X \perp Y \in \mathcal{K}^{*} .
\end{array}\right.
$$

The nonnegativity constraints on $X$ and $Y$ are now expressed in terms a Loewnerian cone $\mathcal{K}$ and its positive dual cone $\mathcal{K}^{*}$. By definition, a Loewnerian cone is the image of the SDP cone

$$
\mathcal{P}_{n}:=\left\{X \in \mathcal{S}_{n}: X \succeq \mathbf{0}\right\}
$$

under some invertible linear endomorphism on $\mathcal{S}_{n}$. Loewnerian cones are not self-dual in general, but they share a number of properties of the SDP cone. For instance, a Loewnerian cone has a similar facial structure as the SDP cone.

\section{Complementarity function approach for solving the SDCP}

A natural strategy for solving (1) is to apply the Semismooth Newton Method (SNM) to the nonlinear system

$$
\left\{\begin{array}{l}
\Phi(X, Y, \lambda)=\mathbf{0} \\
\kappa(X, Y)=\mathbf{0},
\end{array}\right.
$$

where $\kappa: \mathcal{S}_{n} \times \mathcal{S}_{n} \rightarrow \mathcal{S}_{n}$ is a complementarity function for the SDP cone, i.e.,

$$
\kappa(X, Y)=\mathbf{0} \quad \Leftrightarrow \quad \mathbf{0} \preceq X \perp Y \succeq \mathbf{0} .
$$

The function $\kappa$ may not be differentiable. In order to apply the SNM one just needs to ensure that $\kappa$ is globally Lipschitz and semismooth. This requirement is fulfilled if one chooses for instance the Fischer-Burmeister complementarity function

$$
\kappa_{\mathrm{fb}}(X, Y):=X+Y-\left(X^{2}+Y^{2}\right)^{1 / 2}
$$

or the minimum complementarity function

$$
\kappa_{\min }(X, Y):=X-\Pi_{\mathcal{P}_{n}}(X-Y) .
$$

The square root operation $(\cdot)^{1 / 2}$ is understood in the usual matrix sense and $\Pi_{\mathcal{P}_{n}}$ stands for the projection map onto $\mathcal{P}_{n}$. The functions $\kappa_{\mathrm{fb}}$ and $\kappa_{\mathrm{min}}$ are known to be globally Lipschitz and strongly semismooth, see [26] and [27].

For notational convenience we write the nonlinear system (6) in the more compact form

$$
\Psi(z)=\mathbf{0},
$$

where $\Psi: \mathbb{E} \rightarrow \mathbb{W}$ is given by

$$
z=(X, Y, \lambda) \mapsto \Psi(z):=(\Phi(X, Y, \lambda), \kappa(X, Y)) .
$$

Thanks to the assumption (2), the space $\mathbb{W}:=\mathbb{F} \times \mathcal{S}_{n}$ has the same dimension as $\mathbb{E}$. In other words, the system (7) has the same number of equations and unknown variables. We solve the square system (7) with the following Semismooth Newton Method (SNM):

- Initialization. Choose an initial point $z_{0}$ and set $t=0$.

- Iteration. One has a current point $z_{t}$. Pick $M_{t} \in \partial \Psi\left(z_{t}\right)$, find $\Delta z$ such that

$$
M_{t} \Delta z=-\Psi\left(z_{t}\right),
$$

and update $z_{t+1}=z_{t}+\Delta z$. 
The symbol $\partial \Psi(z)$ stands for the Clarke generalized Jacobian of $\Psi$ at $z$ (cf. [8]). Theoretical aspects concerning the rate of convergence of the SNM can be consulted in [23].

Remark 2.1 We keep the SNM running until any of the following situations occur:

$$
\begin{array}{ll}
t=1000 & \text { (lack of convergence), } \\
\operatorname{cond}\left(M_{t}\right) \geq 10^{10} & \text { (ill-conditioning), } \\
\left\|\Psi\left(z_{t}\right)\right\| \leq 10^{-8} & \text { (a solution is found) }
\end{array}
$$

Here, $\operatorname{cond}(M)$ refers to the condition number of a linear map $M$. All our numerical experiments are carried in Window 7 with a processor $3.40 \mathrm{GHz}$ Intel Xeon, Memory(RAM) 8.00 $\mathrm{Gb}$. The codes were implemented with Matlab 7.12.

\subsection{Numerical experiments}

The numerical experiments reported in this section concern the problem of finding a Loewnereigenvalue of a given map $\mathfrak{L} \in \operatorname{End}\left(\mathcal{S}_{n}\right)$. So, the problem at hand is to solve (7) with $z=(X, Y, \lambda)$ and

$$
\Psi(z)=(\mathfrak{L}(X)-\lambda X-Y, \operatorname{tr}(X)-1, \kappa(X, Y)) .
$$

The linearized system (8) takes here the particular form

$$
\begin{gathered}
\mathfrak{L}(\Delta X)-\lambda_{t} \Delta X-\Delta Y-(\Delta \lambda) X_{t}=-\left[\mathfrak{L}\left(X_{t}\right)-\lambda_{t} X_{t}-Y_{t}\right] \\
\operatorname{tr}(\Delta X)=-\left[\operatorname{tr}\left(X_{t}\right)-1\right] \\
E_{t}(\Delta X)+F_{t}(\Delta Y)=-\kappa\left(X_{t}, Y_{t}\right)
\end{gathered}
$$

with $\left(E_{t}, F_{t}\right) \in \partial \kappa\left(X_{t}, Y_{t}\right)$. A convenient way to initialize the SNM for finding a zero of the function (9) is to generate a random Gaussian matrix $\Xi \in \mathcal{S}_{n}$ and set:

$$
\begin{aligned}
X_{0} & =[\operatorname{tr}(\Xi)]^{-1} \Xi, \\
\lambda_{0} & =\left\|X_{0}\right\|^{-2}\left\langle\mathfrak{L}\left(X_{0}\right), X_{0}\right\rangle, \\
Y_{0} & =\mathfrak{L}\left(X_{0}\right)-\lambda_{0} X_{0} .
\end{aligned}
$$

That a random matrix $\Xi \in \mathcal{S}_{n}$ is Gaussian means that the entries $\Xi_{i, j}$ (with $i \leq j$ ) are random variables following a standard normal law.

\subsubsection{Performance of $\kappa_{\mathrm{fb}}$}

As a first choice, we let $\kappa$ be the Fisher-Burmeister complementarity function. For the reader's convenience, we record below a useful lemma that can be found in [18].

Lemma 2.2 Let $A, B \in \mathcal{S}_{n}$ be such that $A^{2}+B^{2}$ is nonsingular. Then $\kappa_{\mathrm{fb}}$ is continuously differentiable at $(A, B)$ and the partial differentials (with respect to $X$ and $Y$ ) are given by

$$
\begin{aligned}
& \left(D_{X} \kappa_{\mathrm{fb}}\right)(A, B)=\mathfrak{I}_{\mathcal{S}_{n}}-\mathcal{L}_{\left[A^{2}+B^{2}\right]^{1 / 2}}^{-1} \circ \mathcal{L}_{A} \\
& \left(D_{Y} \kappa_{\mathrm{fb}}\right)(A, B)=\mathfrak{I}_{\mathcal{S}_{n}}-\mathcal{L}_{\left[A^{2}+B^{2}\right]^{1 / 2}}^{-1} \circ \mathcal{L}_{B} .
\end{aligned}
$$

Here, $\mathfrak{I}_{\mathcal{S}_{n}}$ is the identity map on $\mathcal{S}_{n}$ and

$$
U \in \mathcal{S}_{n} \mapsto \mathcal{L}_{C}(U)=C \bullet U:=(C U+U C) / 2
$$

stands for the Lyapunov operator associated to $C \in \mathcal{S}_{n}$. 
Table 1 SNM applied to (9) with $\kappa=\kappa_{\mathrm{fb}}$. Percentages of success and failure

\begin{tabular}{lrrrr}
\hline & $n=3$ & $n=6$ & $n=9$ & $n=12$ \\
\hline Success (\%) & 96.7 & 99.5 & 99.2 & 97.4 \\
Divergence (\%) & 2.3 & 0.4 & 0.5 & 2.0 \\
Ill-conditioning (\%) & 1.0 & 0.1 & 0.3 & 0.6 \\
\hline
\end{tabular}

\begin{tabular}{lllll}
\hline & $n=3$ & $n=6$ & $n=9$ & $n=12$ \\
\hline NIP & 1.05 & 1.01 & 1.01 & 1.04 \\
CPU & 0.2 & 0.6 & 2.9 & 8.4 \\
\hline
\end{tabular}

Table 2 SNM applied to (9) with $\kappa=\kappa_{\mathrm{fb}}$. Average NIPs and average $\mathrm{CPU}$ times needed for detecting a solution

When $\kappa$ is taken as the Fisher-Burmeister complementarity function, Eq. (12) reads

$$
E_{t}(\Delta X)+F_{t}(\Delta Y)=-\kappa_{\mathrm{fb}}\left(X_{t}, Y_{t}\right)
$$

with $\left(E_{t}, F_{t}\right) \in \partial \kappa_{\mathrm{fb}}\left(X_{t}, Y_{t}\right)$. An explicit formula for computing the Clarke subdifferential of $\kappa_{\mathrm{fb}}$ can be found in [28]. If the matrix $X_{t}^{2}+Y_{t}^{2}$ is nonsingular, then Lemma 2.2 yields

$$
\begin{gathered}
E_{t}=\Im_{\mathcal{S}_{n}}-\mathcal{L}_{C_{t}}^{-1} \circ \mathcal{L}_{X_{t}}, \\
F_{t}=\mathfrak{I}_{\mathcal{S}_{n}}-\mathcal{L}_{C_{t}}^{-1} \circ \mathcal{L}_{Y_{t}},
\end{gathered}
$$

where $C_{t}:=\left[X_{t}^{2}+Y_{t}^{2}\right]^{1 / 2}$. In such a case, (15) takes the form

$$
\Delta X-\mathcal{L}_{C_{t}}^{-1}\left(\mathcal{L}_{X_{t}}(\Delta X)\right)+\Delta Y-\mathcal{L}_{C_{t}}^{-1}\left(\mathcal{L}_{Y_{t}}(\Delta Y)\right)=-\kappa_{\mathrm{fb}}\left(X_{t}, Y_{t}\right) .
$$

By applying $\mathcal{L}_{C_{t}}$ on each side of the above equality and rearranging terms, one gets

$$
\left(X_{t}-\left[X_{t}^{2}+Y_{t}^{2}\right]^{1 / 2}\right) \bullet \Delta X+\left(Y_{t}-\left[X_{t}^{2}+Y_{t}^{2}\right]^{1 / 2}\right) \bullet \Delta Y=\left[X_{t}^{2}+Y_{t}^{2}\right]^{1 / 2} \bullet \kappa_{\mathrm{fb}}\left(X_{t}, Y_{t}\right) .
$$

In our first two experiments (cf. Tables 1,2), the map $\mathfrak{L}$ is generated by means of a random mechanism. To be more precise, we suppose that $\mathfrak{L}$ is Gaussian, i.e., the entries of $\mathfrak{L}$ are independent random variables distributed according to a standard normal law. The "entries" of $\mathfrak{L}$ refer to the entries of the matrix representation of $\mathfrak{L}$ with respect to the canonical basis $\left\{E^{i, j}\right\}_{1 \leq i \leq j \leq n}$ of $\mathcal{S}_{n}$. If $\left\{e_{i}\right\}_{i=1}^{n}$ denotes the canonical basis of $\mathbb{R}^{n}$, then

$$
E^{i, j}:= \begin{cases}e_{i} e_{i}^{T} & \text { if } i=j, \\ \left(e_{i} e_{j}^{T}+e_{j} e_{i}^{T}\right) / \sqrt{2} & \text { if } i \neq j .\end{cases}
$$

The dimension of the space $\operatorname{End}\left(\mathcal{S}_{n}\right)$ is equal to $t_{n}^{2}$, where

$$
t_{n}:=\operatorname{dim}\left(\mathcal{S}_{n}\right)=n(n+1) / 2 .
$$

So, one needs exactly $t_{n}^{2}$ scalars for defining $\mathfrak{L}$. The percentages reported in Table 1 are estimated by working with a sample of $10^{4}$ Gaussian random maps $\mathfrak{L}$. Figures are rounded to one decimal place.

The word "Success" in Table 1 means that the selected random initial point $z_{0}$ has led to a solution, i.e., to a root of (9). Of course, as in any Newton type method, one can perfectly well encounter a situation of failure (divergence or ill-conditioning). As one can see from Table 1, the cases of ill-conditioning and divergence do not occur very often. We mention in passing that we never encountered a situation of nonsmoothness, i.e., at each visited point $z_{t}=\left(X_{t}, Y_{t}, \lambda_{t}\right)$, the matrix $X_{t}^{2}+Y_{t}^{2}$ was always nonsingular. 
In order to find a Loewner-eigenvalue of a given $\mathfrak{L}$, one initial point if often enough. On some occasions, however, one has to run the SNM with more than one initial point. Table 2 displays the expected Number of Initial Points (NIPs) needed for detecting a solution. These figures have been estimated by using a sample of $10^{4}$ Gaussian random maps $\mathfrak{L}$, so they correspond to average values. The expected (or average) CPU time needed for detecting a solution increases of course with $n$.

Remark 2.3 Note that (10)-(12) is a system of $n^{2}+n+1$ equations in the same number of unknown variables. So, the order of the system increases quadratically with $n$. This explains somehow the rapid growth in CPU time reported in Table 2.

The results reported in Table 1 are extremely encouraging. However, one should not be overoptimistic when $\mathfrak{L}$ belongs to some special subsets of the space $\operatorname{End}\left(\mathcal{S}_{n}\right)$. We have found that the percentages of success of the SNM are significatively lower if $\mathfrak{L}$ has for instance the special structure

$$
\mathfrak{L}(X)=A X A+\langle C, X\rangle B
$$

where $A, B, C \in \mathcal{S}_{n}$.

Remark 2.4 By the way, as shown in "Appendix", it is possible to construct a triplet $(A, B, C)$ for which the map (17) has infinitely many Loewner-eigenvalues. Such situation is however unlikely to occur if the matrices $A, B, C$ are randomly generated.

Table 3 has been constructed in the same way as Table 1 , except that now $\mathfrak{L}$ has the special form (17), where $A, B, C \in \mathcal{S}_{n}$ are random Gaussian matrices.

Two comments on Table 3 are in order.

(i) Sometimes the current point $z_{t}=\left(X_{t}, Y_{t}, \lambda_{t}\right)$ is such that $X_{t}^{2}+Y_{t}^{2}$ is singular. As shown in the last row of Table 3 , such situation occurs but it is rather rare. In our opinion, it is not worthwhile to discuss in this paper the sophisticated issue of selecting a pair $\left(E_{t}, F_{t}\right)$ in $\partial \kappa_{\mathrm{fb}}\left(X_{t}, Y_{t}\right)$. Instead of bothering with the computation of the set $\partial \kappa_{\mathrm{fb}}\left(X_{t}, Y_{t}\right)$, we simply use Eq. (16), which makes sense even if $X_{t}^{2}+Y_{t}^{2}$ is singular. The fact of encountering a point of nonsmoothness does not lead necessarily to a situation of failure.

(ii) The instances of failure are essentially due to ill-conditioning, and not to divergence. This phenomenon is reinforced when $n$ increases, see the last column of Table 3. Fixing the ill-conditioning problem induced by the special map (17) is not however the main concern of this work.

In spite of the negative results reported in Table 3, one can still consider the SNM as a viable strategy for computing the Loewner-eigenvalues of the map (17). Of course, one must accept the possibility of initializing the SNM with a large number of initial points. As shown in Table 4, the expected NIPs needed for detecting a solution grow rapidly with $n$.

Table 3 SNM applied to (9) with $\kappa=\kappa_{\mathrm{fb}}$. The map $\mathfrak{L}$ has the special form (17)

\begin{tabular}{lcccc}
\hline & $n=3$ & $n=6$ & $n=9$ & $n=12$ \\
\hline Success (\%) & 89.9 & 23.9 & 4.3 & 0.5 \\
Divergence (\%) & 6.6 & 0.8 & 0.1 & 0.0 \\
Ill-conditioning (\%) & 3.5 & 75.3 & 95.6 & 99.5 \\
Nonsmoothness (\%) & 3.5 & 4.0 & 2.4 & 1.0 \\
\hline
\end{tabular}


Table 4 SNM applied to (9) with $\kappa=\kappa_{\mathrm{fb}}$. The map $\mathfrak{L}$ has the special form (17)

\begin{tabular}{lllll}
\hline & $n=3$ & $n=6$ & $n=9$ & $n=12$ \\
\hline NIP & 1.14 & 4.48 & 33.07 & 454.20 \\
CPU & 0.5 & 2.2 & 16.2 & 614.0 \\
\hline
\end{tabular}

\subsubsection{Performance of $\kappa_{\min }$}

We now turn the attention to the minimum complementarity function. Equation (12) takes the form

$$
E_{t}(\Delta X)+F_{t}(\Delta Y)=-\kappa_{\min }\left(X_{t}, Y_{t}\right)
$$

with $\left(E_{t}, F_{t}\right) \in \partial \kappa_{\min }\left(X_{t}, Y_{t}\right)$. We shall need following differentiability lemma due to Malick and Sendov [19]. The notation $\mathbb{O}(n)$ stands for the set of orthogonal matrices of order $n$, $\lambda(X)$ refers to the $n$-dimensional vector whose components are the eigenvalues of $X \in \mathcal{S}_{n}$ arranged in nondecreasing order, $\operatorname{Diag}(x)$ is the diagonal matrix whose diagonal entries are the components of $x \in \mathbb{R}^{n}$, and $\odot$ is used to indicate the Hadamard (or componentwise) product. For each $x \in \mathbb{R}^{n}$ such that $\Pi_{i=1}^{n} x_{i} \neq 0$ and $x_{1} \leq \cdots \leq x_{n}$, one defines $\mathcal{B}(x) \in \mathcal{S}_{n}$ by setting

$$
(\mathcal{B}(x))_{i, j}:= \begin{cases}0 & \text { if } i \leq q, j \leq q, \\ 1 & \text { if } i>q, j>q, \\ x_{j} /\left(x_{j}-x_{i}\right) & \text { if } i \leq q, j>q, \\ x_{i} /\left(x_{i}-x_{j}\right) & \text { if } i>q, j \leq q,\end{cases}
$$

where $q$ is the number of negative components of $x$.

Lemma 2.5 [19] The function $\mathcal{M}: \mathcal{S}_{n} \rightarrow \mathbb{R}$, defined by

$$
\mathcal{M}(X):=\min _{Y \preceq \mathbf{0}} \frac{1}{2}\|X-Y\|^{2},
$$

is differentiable and its gradient at $X$ is equal to $\Pi_{\mathcal{P}_{n}}(X)$. Furthermore, $\mathcal{M}$ is twice differentiable at $X$ if and only if $X$ is nonsingular, in which case

$$
D^{2} \mathcal{M}(X)\left(H_{1}, H_{2}\right)=\left\langle\mathcal{B}(\lambda(X)),\left(U^{T} H_{1} U\right) \odot\left(U^{T} H_{2} U\right)\right\rangle,
$$

where $U \in \mathbb{O}(n)$ is such that $X=U \operatorname{Diag}(\lambda(X)) U^{T}$.

Thus, for all nonsingular $X \in \mathcal{S}_{n}$, one has

$$
D^{2} \mathcal{M}(X)\left(H_{1}, H_{2}\right)=\left\langle D \Pi_{\mathcal{P}_{n}}(X)\left(H_{1}\right), H_{2}\right\rangle
$$

and computing $D \Pi_{\mathcal{P}_{n}}(X)(H)$ is nothing but to find the Riesz representation of the linear functional $D^{2} \mathcal{M}(X)(H, \cdot)$. By using the formula (19), one gets

$$
\begin{aligned}
D \Pi_{\mathcal{P}_{n}}(X)(H) & =U\left[\mathcal{B}(\lambda(X)) \odot\left(U^{T} H U\right)\right] U^{T} \\
& =\sum_{1 \leq i, j \leq n}\left\langle\mathcal{B}(\lambda(X)),\left(U^{T} H U\right) \odot\left(U^{T} E^{i, j} U\right)\right\rangle E^{i, j} .
\end{aligned}
$$

For numerical purposes, we rely on the representation (20). 
Table 5 SNM applied to (9) with $\kappa=\kappa_{\min }$

\begin{tabular}{lcccc}
\hline & $n=3$ & $n=6$ & $n=9$ & $n=12$ \\
\hline Success (\%) & 86.7 & 97.6 & 99.3 & 98.6 \\
Divergence (\%) & 12.9 & 2.4 & 0.6 & 1.0 \\
Ill-conditioning (\%) & 0.4 & 0.0 & 0.1 & 0.4 \\
\hline
\end{tabular}

Table 6 SNM applied to (9) with $\kappa=\kappa_{\min }$

\begin{tabular}{lllcc}
\hline & $n=3$ & $n=6$ & $n=9$ & $n=12$ \\
\hline NIP & 1.23 & 1.04 & 1.01 & 1.04 \\
CPU & 0.9 & 2.6 & 13.7 & 67.0 \\
\hline
\end{tabular}

Lemma 2.6 Let $A, B \in \mathcal{S}_{n}$ be such that $A-B$ is nonsingular. The $\kappa_{\min }$ is differentiable at $(A, B)$ and the partial differentials (with respect to $X$ and $Y$ ) are given by

$$
\begin{aligned}
& \left(D_{X} \kappa_{\min }\right)(A, B)=\mathcal{I}_{\mathcal{S}_{n}}-D \Pi_{\mathcal{P}_{n}}(A-B), \\
& \left(D_{Y} \kappa_{\min }\right)(A, B)=D \Pi_{\mathcal{P}_{n}}(A-B) .
\end{aligned}
$$

By exploiting the formulas established in Lemma 2.6, one sees that (18) takes the form

$$
\left[\mathcal{I}_{\mathcal{S}_{n}}-D \Pi_{\mathcal{P}_{n}}\left(X_{t}-Y_{t}\right)\right](\Delta X)+D \Pi_{\mathcal{P}_{n}}\left(X_{t}-Y_{t}\right)(\Delta Y)=-\kappa_{\min }\left(X_{t}, Y_{t}\right) .
$$

whenever $X_{t}-Y_{t}$ is nonsingular. Tables 5 and 6 have been constructed in the same way as Tables 1 and 2, respectively, except that now $\kappa$ is the minimum complementarity function. The map $\mathfrak{L}$ is Gaussianly generated and has no special structure.

As in Table 1, we never encountered a situation of nonsmoothness. Table 5 shows that, in terms of percentages of success, the performance of $\kappa=\kappa_{\min }$ is of the same order as $\kappa=\kappa_{\mathrm{fb}}$. By contrast, Table 6 shows that the CPU time needed by $\kappa=\kappa_{\min }$ is clearly higher than the CPU time needed by $\kappa=\kappa_{\mathrm{fb}}$. The reason is that the computational effort for determining $\left(E_{t}, F_{t}\right)$ in (18) is much higher than in (15).

Remark 2.7 The performance of $\kappa=\kappa_{\min }$ is similar to that of $\kappa=\kappa_{\mathrm{fb}}$, also when $\mathfrak{L}$ has the special structure (17). For avoiding repetitions, we omit writing down the corresponding table.

\subsection{A brief comment on the squaring technique}

The squaring technique is based on the representability of the SDP cone as a cone of squares: $\mathcal{P}_{n}=\left\{U^{2}: U \in \mathcal{S}_{n}\right\}$. One can get rid of the constraints $X \succeq 0$ and $Y \succeq 0$ by writing $X=U^{2}$ and $Y=V^{2}$. With such change of variables, the model (1) takes the form of a smooth system of equations:

$$
\left\{\begin{array}{l}
\Phi\left(U^{2}, V^{2}, \lambda\right)=\mathbf{0}, \\
\left\langle U^{2}, V^{2}\right\rangle=0 .
\end{array}\right.
$$

Unfortunately, the last equality in (21) is at the origin of a certain ill-conditioning in the whole system. Besides, the system (21) is not square. By following a similar strategy as in $[9,10]$, one may shift the attention to a certain "companion" system

$$
\left\{\begin{array}{l}
\Phi\left(U^{2}, V^{2}, \lambda\right)=\mathbf{0}, \\
U \bullet V=\mathbf{0},
\end{array}\right.
$$


which is smooth, square, and usually well-conditioned. It must be observed that (22) is related, but not equivalent, to the original problem (21). More precisely, $\left\langle U^{2}, V^{2}\right\rangle=0$ implies $U \bullet V=\mathbf{0}$, but not conversely. The triplet $(U, V, \lambda)$ is declared a fake solution to (21) if the system (22) holds, but $\left\langle U^{2}, V^{2}\right\rangle \neq 0$.

Example 2.8 Consider the problem of finding a Loewner-eigenvalue of the map $\mathcal{L}$ given by $\mathcal{L}(X)=\operatorname{tr}(X) I_{n}$, where $I_{n}$ is the identity matrix of order $n$. The companion system (22) becomes

$$
\left\{\begin{array}{l}
I_{n}-\lambda U^{2}-V^{2}=\mathbf{0}, \\
\|U\|^{2}-1=0 \\
U \bullet V=\mathbf{0}
\end{array}\right.
$$

One can check that

$$
(U, V, \lambda)=\left(\frac{-e_{1} e_{1}^{T}+e_{n} e_{n}^{T}}{\sqrt{2}}, \sum_{i=1}^{n} e_{i} e_{n+1-i}^{T}, 0\right)
$$

is a fake solution. Indeed, (24) solves the system (23), but

$$
\left\langle U^{2}, V^{2}\right\rangle=\left\langle\frac{e_{1} e_{1}^{T}+e_{n} e_{n}^{T}}{2}, I_{n}\right\rangle=1 .
$$

If one considers a map $\mathfrak{L} \in \operatorname{End}\left(\mathcal{S}_{n}\right)$ that is randomly generated according to a Gaussian distribution and applies the classical Newton method to the companion system

$$
\left\{\begin{array}{l}
\mathfrak{L}\left(U^{2}\right)-\lambda U^{2}-V^{2}=\mathbf{0}, \\
\|U\|^{2}-1=0, \\
U \bullet V=\mathbf{0},
\end{array}\right.
$$

then one observes experimentally that, in case of convergence, one always obtains a triplet $(U, V, \lambda)$ that is not a fake solution, but a true one. In other words, the delivery of a fake solution is a rather exceptional event. Instances of ill-conditioning in (25) can be observed from time to time, but not frequently. On the negative side, our numerical tests show that Newton's method applied to (25) requires a very careful selection of the initial point in order to ensure convergence. In view of this observation, it is reasonable to introduce a suitable globalization technique as recommended by some authors.

\section{On Loewnerian cones}

In what follows, $\mathbb{G} \mathbb{L}\left(\mathcal{S}_{n}\right)$ stands for the group of invertible linear endomorphisms on $\mathcal{S}_{n}$, i.e.,

$$
\mathbb{G L}\left(\mathcal{S}_{n}\right):=\left\{\mathfrak{F} \in \operatorname{End}\left(\mathcal{S}_{n}\right): \mathfrak{F} \text { is invertible }\right\} .
$$

The next definition concerns a class of closed convex cones that are somewhat similar to $\mathcal{P}_{n}$.

Definition 3.1 A closed convex cone $\mathcal{K}$ in $\mathcal{S}_{n}$ is Loewnerian if it representable as

$$
\mathcal{K}=\{\mathfrak{F}(U): U \succeq \mathbf{0}\}
$$

for some $\mathfrak{F} \in \mathbb{G} \mathbb{L}\left(\mathcal{S}_{n}\right)$. One refers to (26) as the Loewnerian cone induced by $\mathfrak{F}$. 
A Loewnerian cone is nothing but the image of $\mathcal{P}_{n}$ under some invertible linear endomorphism on $\mathcal{S}_{n}$. The map $\mathfrak{F}$ in the representation formula (26) is not unique. In fact,

$$
\left.\begin{array}{l}
\mathfrak{F}_{1}, \mathfrak{F}_{2} \in \mathbb{G} \mathbb{L}\left(\mathcal{S}_{n}\right) \text { induce } \\
\text { the same Loewnerian cone }
\end{array}\right\} \Leftrightarrow\left(\mathfrak{F}_{2}^{-1} \circ \mathfrak{F}_{1}\right)\left(\mathcal{P}_{n}\right)=\mathcal{P}_{n} .
$$

Since $\mathcal{P}_{n}$ is proper and nonpolyhedral, so is any Loewnerian cone. A closed convex cone is called proper if it is pointed and solid. A Loewnerian cone can also be represented in the "inverse image" form

$$
\mathcal{K}=\left\{X \in \mathcal{S}_{n}: \mathfrak{G}(X) \succeq \mathbf{0}\right\}
$$

for some $\mathfrak{G} \in \mathbb{G} \mathbb{L}\left(\mathcal{S}_{n}\right)$. One passes from (26) to the dual representation (27) by taking $\mathfrak{G}$ as the inverse of $\mathfrak{F}$. Conversely, one passes from (27) to the primal representation (26) by taking $\mathfrak{F}$ as the inverse of $\mathfrak{G}$. The dual of a Loewnerian cone is a Loewnerian cone. Indeed, for all $\mathfrak{F} \in \mathbb{G} \mathbb{L}\left(\mathcal{S}_{n}\right)$, one has

$$
\{\mathfrak{F}(U): U \succeq \mathbf{0}\}^{*}=\left\{\mathfrak{F}^{-T}(V): V \succeq \mathbf{0}\right\}
$$

with $\mathfrak{F}^{-T}$ standing for the adjoint map of the inverse of $\mathfrak{F}$. The following proposition concerns the facial structure of Loewnerian cones.

Proposition 3.2 Let $\mathcal{K}$ be a Loewnerian cone induced by $\mathfrak{F} \in \mathbb{G} L\left(\mathcal{S}_{n}\right)$.

(a) Each face of $\mathcal{K}$ is an exposed face. Furthermore, $\{\operatorname{dim}(\mathcal{M}): \mathcal{M}$ face of $\mathcal{K}\}=$ $\left\{t_{1}, \ldots, t_{n}\right\}$, where $t_{k}:=k(k+1) / 2$ stands for the $k$ th triangular number.

(b) $\mathcal{M}$ is a $t_{k}$-dimensional face of $\mathcal{K}$ if and only if $\mathcal{M}=\{\mathfrak{F}(U): U \succeq \mathbf{0}, \operatorname{Im} U \subseteq L\}$ for some linear subspace $L \subseteq \mathbb{R}^{n}$ of dimension $k$.

Proof As an application of [4, Theorem 5], one sees that the transformation

$$
\begin{aligned}
2^{\mathcal{S}_{n}} & \stackrel{\mathfrak{F}_{\sharp}}{\longrightarrow} 2^{\mathcal{S}_{n}} \\
\mathfrak{F}_{\sharp}(\mathcal{E}) & :=\{\mathfrak{F}(U): U \in \mathcal{E}\}
\end{aligned}
$$

is a bijection between the faces of $\mathcal{P}_{n}$ and the faces of $\mathcal{K}$. One can also check that

$$
\operatorname{dim}\left[\mathfrak{F}_{\sharp}(\mathcal{E})\right]=\operatorname{dim}(\mathcal{E})
$$

for any face $\mathcal{E}$ of $\mathcal{P}_{n}$. The rest of the proof is a matter of recalling the well known facial structure of $\mathcal{P}_{n}$, see for instance [5, Chapter II.12] or [12, Section 4.2.2].

A proper cone $\mathcal{K}$ in $\mathcal{S}_{n}$ is called rotund (cf. [25]) if every face of $\mathcal{K}$, other than $\mathcal{K}$ itself and $\{\mathbf{0}\}$, is a half-line. Rotund cones are often times referred to as strictly convex cones because they are characterized by the strict convexity condition

$$
X_{1}, X_{2} \in \mathcal{K} \text { not collinear } \Longrightarrow X_{1}+X_{2} \in \operatorname{int}(\mathcal{K}) .
$$

By definition, a proper cone $\mathcal{K}$ in $\mathcal{S}_{n}$ is smooth if its dual is rotund.

Corollary 3.3 A Loewnerian cone in $\mathcal{S}_{n}$, with $n \geq 3$, is neither rotund nor smooth.

Proof Suppose that $n \geq 3$. A rotund cone in $\mathcal{S}_{n}$ does not have a face of dimension $t_{2}=$ 3. Hence, it cannot be Loewnerian by Proposition 3.2(a). A smooth cone in $\mathcal{S}_{n}$ cannot be Loewnerian either, because its dual is rotund. 


\subsection{Complementarity relative to Loewnerian cones}

Consider the general complementarity problem (5) with $\mathcal{K}$ being a Loewnerian cone induced by $\mathfrak{F}$. Thanks to the duality formula (28) and the fact that

$$
\left\langle\mathfrak{F}(U), \mathfrak{F}^{-T}(V)\right\rangle=\langle U, V\rangle
$$

for all $U, V \in \mathcal{S}_{n}$, the model (5) can be written in the equivalent form

$$
\left\{\begin{array}{l}
\Phi\left(\mathfrak{F}(U), \mathfrak{F}^{-T}(V), \lambda\right)=\mathbf{0}, \\
\mathbf{0} \preceq U \perp V \succeq \mathbf{0} .
\end{array}\right.
$$

The latter complementarity problem has of course the same structure as (1). We are then back to the context of Sect.2. One can solve (30) by using for instance the complementarity function technique with the Fisher-Burmeister function $\kappa_{\mathrm{fb}}$. Another option is to use the complementarity function technique directly on (5). As complementarity function for the Loewnerian cone $\mathcal{K}$ one can use for instance

$$
\widehat{\kappa}_{\mathrm{fb}}(X, Y):=\kappa_{\mathrm{fb}}\left(\mathfrak{F}^{-1}(X), \mathfrak{F}^{T}(Y)\right) .
$$

Example 3.4 Consider the problem of finding a real $\lambda$ such that the system

$$
\mathcal{K} \ni X \perp(\mathfrak{L}(X)-\lambda X) \in \mathcal{K}^{*}
$$

has a nonzero solution $X \in \mathcal{S}_{n}$. If $\mathcal{K}$ is a Loewnerian cone induced by $\mathfrak{F}$, then everything boils down to solve the nonlinear system

$$
\left\{\begin{array}{l}
\mathfrak{L}(\mathfrak{F}(U))-\lambda \mathfrak{F}(U)-\mathfrak{F}^{-T}(V)=\mathbf{0} \\
\operatorname{tr}(\mathfrak{F}(U))-1=0 \\
\kappa_{\mathrm{bf}}(U, V)=\mathbf{0}
\end{array}\right.
$$

Alternatively, one can work with the original variables $X$ and $Y$ :

$$
\left\{\begin{array}{l}
\mathfrak{L}(X)-\lambda X-Y=\mathbf{0}, \\
\operatorname{tr}(X)-1=0 \\
\widehat{\kappa}_{\mathrm{fb}}(X, Y)=\mathbf{0} .
\end{array}\right.
$$

3.2 Examples of Loewnerian cones and counter-examples

We now dress a short list of interesting proper cones that are Loewnerian. We do not claim that all these cones are relevant in the theory of complementarity problems, but it is reasonable to get acquainted with these examples just for the sake of academic knowledge. We start by stating a trivial but useful lemma.

Lemma 3.5 Let $C, B \in \mathcal{S}_{n}$ be such that $\langle C, B\rangle \neq 1$. Then the map $\mathfrak{G}: \mathcal{S}_{n} \rightarrow \mathcal{S}_{n}$ defined by

$$
\mathfrak{G}(X)=\langle C, X\rangle B-X
$$

is invertible and its inverse $\mathfrak{F}: \mathcal{S}_{n} \rightarrow \mathcal{S}_{n}$ is given by

$$
\mathfrak{F}(U)=\frac{\langle C, U\rangle}{\langle C, B\rangle-1} B-U .
$$


Proof For all $U \in \mathcal{S}_{n}$, the matrix equation $\langle C, X\rangle B-X=U$ admits a unique solution $X \in \mathcal{S}_{n}$. Indeed, by taking the inner product with respect to $C$ one gets

$$
\langle C, X\rangle(\langle C, B\rangle-1)=\langle C, U\rangle .
$$

From here one derives $\langle C, X\rangle$ as a function of $U$ and then one finds the solution $X$ itself.

If $X, B$ are symmetric matrices with $B$ positive definite, then $\lambda_{\max }(X, B)$ denotes the largest real $\lambda$ for which $\operatorname{det}(X-\lambda B)=0$. When $B$ is the identity matrix one simply writes $\lambda_{\max }(X)$.

Proposition 3.6 Let $C, B \in \mathcal{S}_{n}$ with $B$ positive definite. The condition $\langle C, B\rangle \neq 1$ is necessary and sufficient for the closed convex cone

$$
\mathcal{K}=\left\{X \in \mathcal{S}_{n}: \lambda_{\max }(X, B) \leq\langle C, X\rangle\right\}
$$

to be Loewnerian.

Proof Let $\mathfrak{G}: \mathcal{S}_{n} \rightarrow \mathcal{S}_{n}$ be given by (31). One has

$$
\begin{aligned}
\mathfrak{G}(X) \succeq \mathbf{0} & \Leftrightarrow\langle C, X\rangle B \succeq X \\
& \Leftrightarrow\langle u, X u\rangle \leq\langle C, X\rangle\langle u, B u\rangle \text { for all } u \in \mathbb{R}^{n} \\
& \Leftrightarrow \lambda_{\max }(X, B) \leq\langle C, X\rangle .
\end{aligned}
$$

In other words, $\mathcal{K}$ is representable as in (27). If $\langle C, B\rangle \neq 1$, then $\mathfrak{G}$ is invertible by Lemma 3.5, and therefore $\mathcal{K}$ is Loewnerian. If $\langle C, B\rangle=1$, then $\mathbb{R}(B)=\operatorname{Ker}(\mathfrak{G}) \subseteq \mathcal{K}$. Hence, $\mathcal{K}$ is not pointed because it contains the line generated by $B$.

Remark 3.7 Suppose that $\langle C, B\rangle \neq 1$. The Loewnerian cone (32) is not self-dual in general. However, its dual

$$
\mathcal{K}^{*}=\left\{Y \in \mathcal{S}_{n}: \lambda_{\max }(Y, C) \leq \frac{\langle B, Y\rangle}{\langle C, B\rangle-1}\right\}
$$

has the same structure as (32).

Corollary 3.8 Let $\alpha \in \mathbb{R} \backslash\{0,1\}$. Then

$$
\begin{aligned}
K & =\left\{X \in \mathcal{S}_{n}: \alpha \lambda_{\max }(X) \leq n^{-1} \operatorname{tr}(X)\right\} \\
K^{*} & =\left\{Y \in \mathcal{S}_{n}:(1-\alpha) \lambda_{\max }(Y) \leq n^{-1} \operatorname{tr}(Y)\right\}
\end{aligned}
$$

are mutually dual Loewnerian cones.

Proof One just needs to apply Proposition 3.6 with $B=I_{n}$ and $C=(\alpha n)^{-1} I_{n}$.

Recall that $\lambda(X)=\left(\lambda_{1}(X), \ldots, \lambda_{n}(X)\right)^{T}$ is the vector whose components are the eigenvalues of $X \in \mathcal{S}_{n}$ arranged in nondecreasing order. A spectral proper cone in $\mathcal{S}_{n}$ is a set of the form

$$
\mathcal{K}=\left\{X \in \mathcal{S}_{n}: \lambda(X) \in P\right\},
$$

where $P$ is a permutation invariant proper cone in $\mathbb{R}^{n}$. An interesting family $\left\{\mathcal{K}_{q}^{\uparrow}\right\}_{q=1}^{n}$ of spectral proper cones arising in applications is given by

$$
\mathcal{K}_{q}^{\uparrow}:=\left\{X \in \mathcal{S}_{n}: \sum_{i=1}^{q} \lambda_{i}(X) \geq 0\right\} .
$$


These cones have been studied in [3] and in many other publications. For $q=1$, one gets

$$
\mathcal{K}_{1}^{\uparrow}=\left\{X \in \mathcal{S}_{n}: \lambda_{\min }(X) \geq 0\right\}=\mathcal{P}_{n} .
$$

The choice $q=n-1$ leads to

$$
\mathcal{K}_{n-1}^{\uparrow}=\left\{X \in \mathcal{S}_{n}: \lambda_{\max }(X) \leq \operatorname{tr}(X)\right\},
$$

which is also a Loewnerian cone (cf. Corollary 3.8). The analysis of the case $2 \leq q \leq n-2$ is more involved. The next proposition concerns the choice $q=2$.

Proposition 3.9 Let $n \geq 4$. The cone

$$
\mathcal{K}_{2}^{\uparrow}=\left\{X \in \mathcal{S}_{n}: \lambda_{1}(X)+\lambda_{2}(X) \geq 0\right\}
$$

is not Loewnerian.

Proof The cone $\mathcal{K}_{2}^{\uparrow}$ admits the representation (33) with

$$
P=\left\{x \in \mathbb{R}^{n}: x_{i}+x_{j} \geq 0 \text { for all } 1 \leq i<j \leq n\right\} .
$$

Such set $P$ is a permutation invariant proper cone. Note that $P$ is also polyhedral. In view of [14, Lemma 2.1], the set

$$
E=\left\{x \in P: x_{i}+x_{n-1}=0 \text { for all } i=1, \ldots, n-2\right\}
$$

is a two-dimensional exposed face of $P$. By using such face $E$ and Lewis's facial theorem [16, Theorem 5.1], one can construct a two-dimensional face for $\mathcal{K}_{2}^{\uparrow}$. This fact and Proposition 3.2(a) prove that $\mathcal{K}_{2}^{\uparrow}$ is not Loewnerian.

The cone $\mathcal{K}_{n-2}^{\uparrow}$ is not Loewnerian either, because it is the image of $\mathcal{K}_{2}^{\uparrow}$ under a nonsingular transformation. In fact, one has the following general result.

Proposition 3.10 Let $q \in\{1, \ldots, n-1\}$. Then the linear map

$$
X \in \mathcal{S}_{n} \mapsto \mathfrak{G}(X):=\frac{\operatorname{tr}(X)}{q} I_{n}-X
$$

is a bijection between $\mathcal{K}_{n-q}^{\uparrow}$ and $\mathcal{K}_{q}^{\uparrow}$.

Proof That $\mathfrak{G}$ is invertible is clear from Lemma 3.5. Since

$$
\lambda_{i}(\mathfrak{G}(X))=\frac{\operatorname{tr}(X)}{q}-\lambda_{n-i+1}(X)
$$

for all $i \in\{1, \ldots, n\}$, one has

$$
\sum_{i=1}^{q} \lambda_{i}(\mathfrak{G}(X))=\operatorname{tr}(X)-\sum_{i=1}^{q} \lambda_{n-i+1}(X)=\sum_{i=1}^{n-q} \lambda_{i}(X) .
$$

Hence,

$$
\begin{aligned}
\mathcal{K}_{n-q}^{\uparrow} & =\left\{X \in \mathcal{S}_{n}: \sum_{i=1}^{n-q} \lambda_{i}(X) \geq 0\right\} \\
& =\left\{X \in \mathcal{S}_{n}: \sum_{i=1}^{q} \lambda_{i}(\mathfrak{G}(X)) \geq 0\right\}=\mathfrak{G}^{-1}\left(\mathcal{K}_{q}^{\uparrow}\right) .
\end{aligned}
$$

This completes the proof. 
By a revolution cone in $\mathcal{S}_{n}$ one understands a proper cone of the form

$$
\operatorname{Rev}(s, Y):=\left\{U \in \mathcal{S}_{n}: s\|U\| \leq\langle Y, U\rangle\right\},
$$

where $0<s<1$ and $Y \in \mathcal{S}_{n}$ is such that $\|Y\|=1$. A revolution cone is a particular instance of an ellipsoidal cone. By definition, an ellipsoidal cone in $\mathcal{S}_{n}$ is a set representable as

$$
\mathcal{E}(\mathfrak{D}, B):=\left\{X \in \mathcal{S}_{n}:\|\mathfrak{D}(X)\| \leq\langle B, X\rangle\right\},
$$

where $B \in \mathcal{S}_{n}$ and $\mathfrak{D} \in \mathbb{G} \mathbb{L}\left(\mathcal{S}_{n}\right)$ are such that $\left\|\mathfrak{D}^{-T}(B)\right\|>1$. The latter inequality ensures that (35) is a proper cone. Whether an ellipsoidal cone in $\mathcal{S}_{n}$ is Loewnerian or not depends on the dimension $n$. The case $n=2$ is somewhat special.

\section{Proposition 3.11 One has:}

(a) A proper cone in $\mathcal{S}_{2}$ is Loewnerian if and only if it is an ellipsoidal cone.

(b) Any ellipsoidal cone in $\mathcal{S}_{n}$, with $n \geq 3$, is not Loewnerian.

Proof One easily sees that (35) satisfies the strict convexity condition (29). Hence, (b) is a consequence of Corollary 3.3. Consider now the particular case $n=2$. Note that

$$
\mathcal{E}(\mathfrak{D}, B)=\left\{\mathfrak{D}^{-1}(U): U \in \operatorname{Rev}\left(\frac{1}{\left\|\mathfrak{D}^{-T} B\right\|}, \frac{\mathfrak{D}^{-T}(B)}{\left\|\mathfrak{D}^{-T} B\right\|}\right)\right\},
$$

i.e., the action of $\mathfrak{D}$ on the ellipsoidal cone (35) produces a revolution cone. So, it is enough to show that a suitable chain

$$
\mathcal{S}_{2} \stackrel{\mathfrak{L}_{1}}{\longrightarrow} \mathbb{R}^{3} \stackrel{\mathfrak{L}_{2}}{\longrightarrow} \mathbb{R}^{3} \stackrel{\mathfrak{L}_{3}}{\longrightarrow} \mathcal{S}_{2}
$$

of linear isomorphisms allows us to pass from a revolution cone to $\mathcal{P}_{2}$. One starts with the standard linear isometry

$$
\mathfrak{L}_{1}\left(\left[\begin{array}{cc}
\alpha & \beta \\
\beta & \gamma
\end{array}\right]\right):=\left[\begin{array}{c}
\alpha \\
\sqrt{2} \beta \\
\gamma
\end{array}\right]
$$

between $\mathcal{S}_{2}$ and $\mathbb{R}^{3}$. The action of $\mathfrak{L}_{1}$ on a revolution cone $\operatorname{Rev}(s, Y)$ in $\mathcal{S}_{2}$ produces a revolution cone

$$
\operatorname{rev}(s, y):=\left\{u \in \mathbb{R}^{3}: s\|u\| \leq y^{T} u\right\}
$$

in $\mathbb{R}^{3}$. Then one constructs a linear invertible map $\mathfrak{L}_{2}: \mathbb{R}^{3} \rightarrow \mathbb{R}^{3}$ that converts $\operatorname{rev}(s, y)$ into the ice-cream cone

$$
\mathbb{L}_{3}:=\left\{x \in \mathbb{R}^{3}:\left[x_{1}^{2}+x_{2}^{2}\right]^{1 / 2} \leq x_{3}\right\} .
$$

Such an $\mathfrak{L}_{2}$ clearly exists. Finally, one constructs a linear isomorphism $\mathfrak{L}_{3}: \mathbb{R}^{3} \rightarrow \mathcal{S}_{2}$ that converts $\mathbb{L}_{3}$ into $\mathcal{P}_{2}$. The explicit form of $\mathfrak{L}_{3}$ can be found in [12, Section 2.6] or in [21, Chapter 2.5].

The next proposition concerns two classes of closed convex cones arising in the stability analysis of dynamical systems.

Proposition 3.12 Consider a matrix $A \in \mathbb{M}_{n}$ with possible complex eigenvalues $\mu_{1}, \ldots, \mu_{n}$. Then

$$
\begin{aligned}
& \mathcal{K}_{\text {lyap }}:=\left\{X \in \mathcal{S}_{n}: A X+X A^{T} \succeq \mathbf{0}\right\}, \\
& \mathcal{K}_{\text {stein }}:=\left\{X \in \mathcal{S}_{n}: X \succeq A^{T} X A\right\}
\end{aligned}
$$


are Loewnerian cones under the respective assumptions

$$
\begin{gathered}
\mu_{i}+\mu_{j} \neq 0 \text { for all } i, j \in\{1, \ldots, n\}, \\
\mu_{i} \mu_{j} \neq 1 \text { for all } i, j \in\{1, \ldots, n\} .
\end{gathered}
$$

Proof The cones (36) and (37) are representable as in (27) with $\mathfrak{G}$ given respectively by

$$
\begin{aligned}
\mathfrak{G}_{\text {lyap }}(X) & :=A X+X A^{T}, \\
\mathfrak{G}_{\text {stein }}(X) & :=X-A^{T} X A .
\end{aligned}
$$

As established in [15], the spectral condition (38) implies the invertibility of the Lyapunov operator $\mathfrak{G}_{\text {lyap }}: \mathcal{S}_{n} \rightarrow \mathcal{S}_{n}$, whereas (39) implies the invertibility of the Stein operator $\mathfrak{G}_{\text {stein }}: \mathcal{S}_{n} \rightarrow \mathcal{S}_{n}$.

\section{By way of application: finding the nearest Euclidean distance matrix}

A square matrix $A$ of order $m$ is an Euclidean distance matrix (EDM) if there are points $\left\{p_{i}\right\}_{i=1}^{m}$ in some Euclidean space $\mathbb{R}^{d}$ such that

$$
a_{i j}=\left\|p_{i}-p_{j}\right\|_{2}^{2} \text { for all } i, j \in\{1, \ldots, m\},
$$

where $\|\cdot\|_{2}$ is the usual Euclidean norm on $\mathbb{R}^{d}$. The EDMs of order $m$ form a proper cone in the space

$$
\mathcal{S}_{m}^{\bullet}:=\left\{X \in \mathcal{S}_{m}: \operatorname{diag}(X)=\mathbf{0}\right\},
$$

where $\operatorname{diag}(X)$ denotes the vector whose components are the diagonal entries of $X$. There is a rich literature devoted to the analysis and applications of the cone

$$
\mathcal{E}_{m}:=\left\{A \in \mathcal{S}_{m}^{\bullet}: A \text { is an EDM }\right\}
$$

The following proposition exploits the fact that $\mathcal{S}_{n+1}^{\bullet}$ has the same dimension as $\mathcal{S}_{n}$.

Proposition 4.1 Let $\phi$ be a linear isomorphism between $\mathcal{S}_{n+1}^{\bullet}$ and $\mathcal{S}_{n}$. Then

$$
\mathcal{K}=\left\{\phi(A): A \in \mathcal{E}_{n+1}\right\}
$$

is a Loewnerian cone.

Proof There exists a linear isomorphism $\mathfrak{L}: \mathcal{S}_{n} \rightarrow \mathcal{S}_{n+1}^{\bullet}$ such that

$$
\mathcal{E}_{n+1}=\{\mathfrak{L}(U): U \succeq \mathbf{0}\} .
$$

As shown in [1, Theorem 3.2], one may consider for instance

$$
\mathfrak{L}(U):=\left[\begin{array}{cc}
0 & {[\operatorname{diag}(U)]^{T}} \\
\operatorname{diag}(U) & \mathfrak{B}(U)-2 U
\end{array}\right],
$$

where

$$
\mathfrak{B}(U):=[\operatorname{diag}(U)] \mathbf{1}_{n}^{T}+\mathbf{1}_{n}[\operatorname{diag}(U)]^{T}
$$

and $\mathbf{1}_{n}$ is the $n$-dimensional vector of ones. Hence, (40) is a Loewnerian cone induced by the composition $\mathfrak{F}=\phi \circ \mathfrak{L}$. 
Remark 4.2 The dual of (40) is the Loewnerian cone induced by $\phi^{-T} \circ \mathfrak{L}^{-T}$. A matter of computation shows that $\mathfrak{L}^{-T}: \mathcal{S}_{n} \rightarrow \mathcal{S}_{n+1}^{\bullet}$ is given by

$$
\mathfrak{L}^{-T}(V)=\frac{1}{2}\left[\begin{array}{cc}
0 & \left(V \mathbf{1}_{n}\right)^{T} \\
V \mathbf{1}_{n} & -V^{\diamond}
\end{array}\right],
$$

where $V^{\diamond}$ is derived from $V$ by setting zero to the diagonal entries.

An interesting problem of numerical linear algebra addressed in [1,11,22] is this: Given a matrix $C \in \mathcal{S}_{n+1}^{\bullet}$ generated by a random mechanism or obtained as outcome of some physical experiment, one wishes to determine

$$
\Pi_{\mathcal{E}_{n+1}}(C):=\text { projection of } C \text { onto } \mathcal{E}_{n+1} .
$$

This problem is handled in $[1,11]$ by expressing $\mathcal{E}_{n+1}$ as intersection of two "simpler" cones and by applying an alternating projection algorithm. We use here a completely different approach:

- Firstly, we introduce a linear isometry $\phi: \mathcal{S}_{n+1}^{\bullet} \rightarrow \mathcal{S}_{n}$ and shift the attention to the Loewnerian cone $\mathcal{K}=\phi\left(\mathcal{E}_{n+1}\right)$. Since $\phi$ is not merely a linear isomorphism, but also a linear isometry, one has

$$
\Pi_{\mathcal{E}_{n+1}}(C)=\phi^{T}\left(\Pi_{\mathcal{K}}(A)\right)
$$

with $A:=\phi(C)$. Indeed,

$$
\begin{aligned}
\left\|\phi^{T}\left(\Pi_{\mathcal{K}}(A)\right)-C\right\| & =\left\|\Pi_{\mathcal{K}}(A)-A\right\|=\min _{X \in \mathcal{K}}\|X-A\| \\
& =\min _{W \in \mathcal{E}_{n+1}}\|\phi(W)-A\|=\min _{W \in \mathcal{E}_{n+1}}\|W-C\| .
\end{aligned}
$$

- Secondly, in order to find the projection of $A$ onto $\mathcal{K}$, we solve the complementarity problem

$$
\mathcal{K} \ni X \perp(X-A) \in \mathcal{K}^{*}
$$

by using the SNM and a suitable complementarity function.

We now explain the details. As linear isometry $\phi: \mathcal{S}_{n+1}^{\bullet} \rightarrow \mathcal{S}_{n}$ we consider the map

$$
\phi\left(\left[\begin{array}{cc}
0 & b^{T} \\
b & M
\end{array}\right]\right)=M+\sqrt{2} \operatorname{Diag}(b),
$$

whose adjoint is given by

$$
\phi^{T}(Z)=\left[\begin{array}{cc}
0 & \frac{1}{\sqrt{2}}[\operatorname{diag}(Z)]^{T} \\
\frac{1}{\sqrt{2}} \operatorname{diag}(Z) & Z^{\diamond}
\end{array}\right] .
$$

Hence, $\mathcal{K}=\phi\left(\mathcal{E}_{n+1}\right)$ is the Loewnerian cone induced by

$$
\mathfrak{F}(U)=\mathfrak{B}(U)-2 U+\sqrt{2} \operatorname{Diag}(\operatorname{diag}(U)) .
$$

A matter of computation shows that

$$
\begin{aligned}
\mathfrak{F}^{-1}(X) & =(1 / \sqrt{8}) \mathfrak{B}(X)-(1 / 2) X^{\diamond}, \\
\mathfrak{F}^{T}(Y) & =\operatorname{Diag}\left(\sqrt{2} \operatorname{diag}(Y)+2 Y^{\diamond} \mathbf{1}_{n}\right)-2 Y^{\diamond}, \\
\mathfrak{F}^{-T}(V) & =(1 / \sqrt{2}) \operatorname{Diag}\left(V \mathbf{1}_{n}\right)-(1 / 2) V^{\diamond} .
\end{aligned}
$$


Table 7 SNM applied to (42)

\begin{tabular}{lcccc}
\hline & $n=25$ & $n=50$ & $n=75$ & $n=100$ \\
\hline Success (\%) & 100 & 100 & 100 & 100 \\
Divergence (\%) & 0 & 0 & 0 & 0 \\
Ill-conditioning (\%) & 0 & 0 & 0 & 0 \\
CPU (\%) & 5.9 & 79.6 & 217.6 & 839.1 \\
\hline
\end{tabular}

Table 8 SNM applied to (43)

\begin{tabular}{lcccc}
\hline & $n=25$ & $n=50$ & $n=75$ & $n=100$ \\
\hline Success (\%) & 100 & 100 & 100 & 100 \\
Divergence (\%) & 0 & 0 & 0 & 0 \\
Ill-conditioning (\%) & 0 & 0 & 0 & 0 \\
CPU (\%) & 6.2 & 81.1 & 222.5 & 840.6 \\
\hline
\end{tabular}

One can solve the complementarity problem (41) by applying the SNM to the system

$$
\left\{\begin{array}{l}
X-Y-A=\mathbf{0}, \\
\widehat{\kappa}_{\mathrm{fb}}(X, Y)=\mathbf{0} .
\end{array}\right.
$$

We initialize the algorithm with a random Gaussian matrix $X_{0}$ and $Y_{0}=X_{0}-A$. The complementarity problem (41) can also be solved by applying the SNM to

$$
\left\{\begin{array}{l}
\mathfrak{F}(U)-\mathfrak{F}^{-T}(V)-A=\mathbf{0}, \\
\kappa_{\mathrm{fb}}(U, V)=\mathbf{0} .
\end{array}\right.
$$

By way of initialization, we generate a random Gaussian matrix $\Xi$ and set $U_{0}=\mathfrak{F}^{-1}(\Xi)$ and $V_{0}=\mathfrak{F}^{T}(\Xi-A)$.

Tables 7 and 8 report the performance of the SNM applied to (42) and (43), respectively. The figures displayed in these tables are average values obtained by working with a sample of $10^{2}$ random Gaussian matrices $C$.

Remark 4.3 In a recent paper by Qi [22], the nearest EDM problem is treated by passing to a certain dual optimization problem

$$
\operatorname{minimize}_{z \in \mathbb{R}^{n+1}} \theta(z):=(1 / 2)\left\|\Pi_{\mathcal{Q}_{n+1}}[C+\operatorname{Diag}(z)]\right\|^{2},
$$

where

$$
\mathcal{Q}_{n+1}:=\left\{A \in \mathcal{S}_{n+1}:\langle x, A x\rangle \geq 0 \text { when }\left\langle\mathbf{1}_{n+1}, x\right\rangle=0\right\} .
$$

Note that the optimization problem (44) is unconstrained. The approach followed in [22] consists in using the SNM to find a root of the gradient map

$$
\nabla \theta(z):=\operatorname{diag}\left(\Pi_{\mathcal{Q}_{n+1}}[C+\operatorname{Diag}(z)]\right) .
$$

Once a root $\bar{z}$ has been found, then one evaluates

$$
\Pi_{\mathcal{E}_{n+1}}(C)=\Pi_{\mathcal{Q}_{n+1}}(C+\operatorname{Diag}(\bar{z})) .
$$

Projecting onto $\mathcal{Q}_{n+1}$ offers no difficulty. 


\section{By way of conclusion}

This work shows that the SDCP can be solved efficiently by applying the SNM to the system (6). The best results in terms of CPU time are obtained with the help of the Fisher-Burmeister complementarity function $\kappa_{\mathrm{fb}}$. Some comments concerning the genesis of this work and the related literature are appropriate.

1. The idea of applying the SNM to a cone-constrained eigenvalue problem was considered for the first time by Adly and Seeger [2]. The specific problem treated in [2] is that of finding Pareto-eigenvalues in a given matrix $A$. This amounts to solve a complementarity problem

$$
\left\{\begin{array}{l}
A x-\lambda x-y=\mathbf{0}, \\
\left\langle\mathbf{1}_{n}, x\right\rangle-1=0, \\
\mathbf{0} \leq x \perp y \geq \mathbf{0}
\end{array}\right.
$$

involving the usual cone $\mathbb{R}_{+}^{n}$. Although the model (3) looks similar to (45), there are important differences. To start with, (3) leads to a square system with $n^{2}+n+1$ equations, whereas (45) leads to a square system with only $2 n+1$ equations. Hence, the involved dimensions are not of the same order. Secondly, the nonnegative orthant is a polyhedral cone, whereas the SDP cone is non-polyhedral. As a consequence of this fact, a matrix $A$ has always a finite number of Pareto-eigenvalues, whereas a linear endomorphism $\mathfrak{L}$ on $\mathcal{S}_{n}$ may perfectly well have a continuum of Loewner-eigenvalues (cf.Proposition 5.1). In other words, the solution sets to (3) and (45) are structurally different. Thirdly, the squaring technique

$$
\begin{aligned}
& x=u^{[2]}:=u \odot u, \\
& y=v^{[2]}:=v \odot v
\end{aligned}
$$

based on the Hadamard product leads to a companion system

$$
\left\{\begin{array}{l}
A u^{[2]}-\lambda u^{[2]}-v^{[2]}=\mathbf{0}, \\
\|u\|^{2}-1=0, \\
u \odot v=\mathbf{0},
\end{array}\right.
$$

that is free of fake solutions. By contrast, the companion system (25) associated to (3) may well admit fake solutions. In short, a complementarity problem relative to the SDP cone differs substantially from a complementarity problems relative to the nonnegative orthant.

2. The numerical resolution of Example 1.1 is treated here for the first time. Example 1.1 has been the driving motivation behind our work. By contrast, Example 1.2 is mentioned just to illustrate that the SDCP model (1) covers a wide variety of applications. The literature dealing with the optimization problem (4) is quite extense and comparing different methods for solving (4) is beyond the scope of this work.

Acknowledgments Both authors would like to thank the referees for meticulous reading of the manuscript and for several suggestions that improved the presentation. 


\section{Appendix}

Let $\sigma\left(\mathfrak{L}, \mathcal{P}_{n}\right)$ denote the set of Loewner-eigenvalues of a linear map $\mathfrak{L}: \mathcal{S}_{n} \rightarrow \mathcal{S}_{n}$. Such set is called the Loewner-spectrum of $\mathfrak{L}$. The following proposition displays a map $\mathfrak{L}$ whose Loewner-spectrum is a set of infinite cardinality.

Proposition 5.1 Let $\mathfrak{L}: \mathcal{S}_{n} \rightarrow \mathcal{S}_{n}$ be given by $\mathfrak{L}(X)=\langle C, X\rangle I_{n}$, where $C \succeq \mathbf{0}$. Then

$$
\left[\lambda_{\min }(C), \lambda_{\max }(C)\right] \subseteq \sigma\left(\mathfrak{L}, \mathcal{P}_{n}\right)=\bigcup_{r=1}^{n}\left[f_{r}(C), g_{r}(C)\right],
$$

where $f_{r}(C)$ and $g_{r}(C)$ indicate respectively the sum of the $r$ smallest and the sum of the $r$ largest eigenvalues of $C$.

Proof Assume that $C \neq \mathbf{0}$, otherwise (47) holds trivially. A scalar $\lambda$ belongs to $\sigma\left(\mathfrak{L}, \mathcal{P}_{n}\right)$ if and only if there exists $X \in \mathcal{S}_{n}$ such that

$$
\left\{\begin{array}{l}
X \succeq \mathbf{0}, \operatorname{tr}(X)=1, \\
\langle C, X\rangle I_{n} \succeq \lambda X, \\
\langle C, X\rangle=\lambda\|X\|^{2} .
\end{array}\right.
$$

Hence,

$$
\sigma\left(\mathfrak{L}, \mathcal{P}_{n}\right)=\left\{\|X\|^{-2}\langle C, X\rangle: X \in \Omega\right\},
$$

where $\Omega$ stands for the set of matrices $X \in \mathcal{S}_{n}$ satisfying

$$
\begin{gathered}
X \succeq \mathbf{0}, \operatorname{tr}(X)=1, \\
\langle C, X\rangle\left(\|X\|^{2}-\lambda_{\max }(X)\right) \geq 0 .
\end{gathered}
$$

Under (49), the inequality (50) can be written as an equality. The set on the right-hand side of (48) remains unchanged if one uses

$$
\Omega_{0}:=\left\{X \in \mathcal{S}_{n}: X \succeq \mathbf{0}, \operatorname{tr}(X)=1, \lambda_{\max }(X)=\|X\|^{2}\right\}
$$

instead of $\Omega$. On the other hand, one can check that

$$
\Omega_{0}=\bigcup_{r=1}^{n}\left\{r^{-1} Q Q^{T}: Q \in \mathcal{O}(n, r)\right\},
$$

where $Q \in \mathcal{O}(n, r)$ indicates that $Q$ is matrix of size $n \times r$ such that $Q^{T} Q=I_{r}$. Hence,

$$
\sigma\left(\mathfrak{L}, \mathcal{P}_{n}\right)=\bigcup_{r=1}^{n}\left\{\left\langle C, Q Q^{T}\right\rangle: Q \in \mathcal{O}(n, r)\right\} .
$$

Note that $\left\langle C, Q Q^{T}\right\rangle$ ranges over the interval $\left[f_{r}(C), g_{r}(C)\right]$ as the variable $Q$ ranges over $\mathcal{O}(n, r)$.

\section{References}

1. Al-Homidan, S., Wolkowicz, H.: Approximate and exact completion problems for Euclidean distance matrices using semidefinite programming. Linear Algebra Appl. 406, 109-141 (2005) 
2. Adly, S., Seeger, A.: A nonsmooth algorithm for cone-constrained eigenvalue problems. Comput. Optim. Appl. 49, 299-318 (2011)

3. Azé, D., Hiriart-Urruty, J.-B.: Optimal Hoffman-type estimates in eigenvalue and semidefinite inequality constraints. J. Glob. Optim. 24, 133-147 (2002)

4. Barker, G.P., Carlson, D.: Cones of diagonally dominant matrices. Pac. J. Math. 57, 15-32 (1975)

5. Barvinok, A.: A Course in Convexity. American Mathematical Society, Providence (2002)

6. Borsdorf, R., Higham, N.J.: A preconditioned Newton algorithm for the nearest correlation matrix. IMA J. Numer. Anal. 30, 94-107 (2010)

7. Chen, X., Tseng, P.: Non-interior continuation methods for solving semidefinite complementarity problems. Math. Program. Ser. A 95, 431-474 (2003)

8. Clarke, F.H.: Optimization and Nonsmooth Analysis. Wiley, New York (1983)

9. Gajardo, P., Seeger, A.: Reconstructing a matrix from a partial sampling of Pareto eigenvalues. Comput. Optim. Appl. 51, 1119-1135 (2012)

10. Gajardo, P., Seeger, A.: Solving inverse cone-constrained eigenvalue problems. Numerische Math. 123, 309-331 (2013)

11. Glunt, W., Hayden, T.L., Hong, S., Wells, J.: An alternating projection algorithm for computing the nearest Euclidean distance matrix. SIAM J. Matrix Anal. Appl. 11, 589-600 (1990)

12. Hiriart-Urruty, J.B., Malick, J.: A fresh variational-analysis look at the positive semidefinite matrices world. J. Optim. Theory Appl. 153, 551-577 (2012)

13. Isac, G., Bulavsky, V.A., Kalashnikov, V.V.: Complementarity, Equilibrium, Efficiency and Economics. Kluwer, Dordrecht (2002)

14. Kim, N.T.B., Luc, D.T.: Normal cones to a polyhedral convex set and generating efficient faces in linear multiobjective programming. Acta Math. Vietnam. 25, 101-124 (2000)

15. Lancaster, P., Rodman, L.: Algebraic Riccati Equations. Oxford University Press, New York (1995)

16. Lewis, A.S.: Eigenvalue-constrained faces. Linear Algebra Appl. 269, 159-181 (1998)

17. Li, Q., Li, D., Qi, H.: Newton's method for computing the nearest correlation matrix with a simple upper bound. J. Optim. Theory Appl. 147, 546-568 (2010)

18. Liu, Y.J., Zhang, L.W., Wang, Y.H.: Some properties of a class of merit functions for symmetric cone complementarity problems. Asia-Pac. J. Oper. Res. 23, 473-495 (2006)

19. Malick, J., Sendov, H.: Clarke generalized Jacobian of the projection onto the cone of positive semidefinite matrices. Set-Valued Anal. 14, 273-293 (2006)

20. Nishimura, R., Hayashi, S., Fukushima, M.: Semidefinite complementarity reformulation for robust Nash equilibrium problems with Euclidean uncertainty sets. J. Glob. Optim. 53, 107-120 (2012)

21. Pukelsheim, F.: Optimal Design of Experiments. Wiley, New York (1993)

22. Qi, H.D.: A semismooth Newton method for the nearest Euclidean distance matrix problem. SIAM J. Matrix Anal. Appl. 34, 67-93 (2013)

23. Qi, L., Sun, J.: A nonsmooth version of Newton's method. Math. Program. Ser. A 58, 353-367 (1993)

24. Seeger, A.: Eigenvalue analysis of equilibrium processes defined by linear complementarity conditions. Linear Algebra Appl. 292, 1-14 (1999)

25. Stern, R.J., Wolkowicz, H.: Invariant ellipsoidal cones. Linear Algebra Appl. 150, 81-106 (1991)

26. Sun, D., Sun, J.: Semismooth matrix-valued functions. Math. Oper. Res. 27, 150-169 (2002)

27. Sun, D., Sun, J.: Strong semismoothness of the Fischer-Burmeister SDC and SOC complementarity functions. Math. Program. Ser. A 103, 575-581 (2005)

28. Zhang, L., Zhang, N., Pang, L.: Differential properties of the symmetric matrix-valued Fischer-Burmeister function. J. Optim. Theory Appl. 153, 436-460 (2012)

29. Zhou, Y., Gowda, M.S.: On the finiteness of the cone spectrum of certain linear transformations on Euclidean Jordan algebras. Linear Algebra Appl. 431, 772-782 (2009) 